\title{
NEWS
}

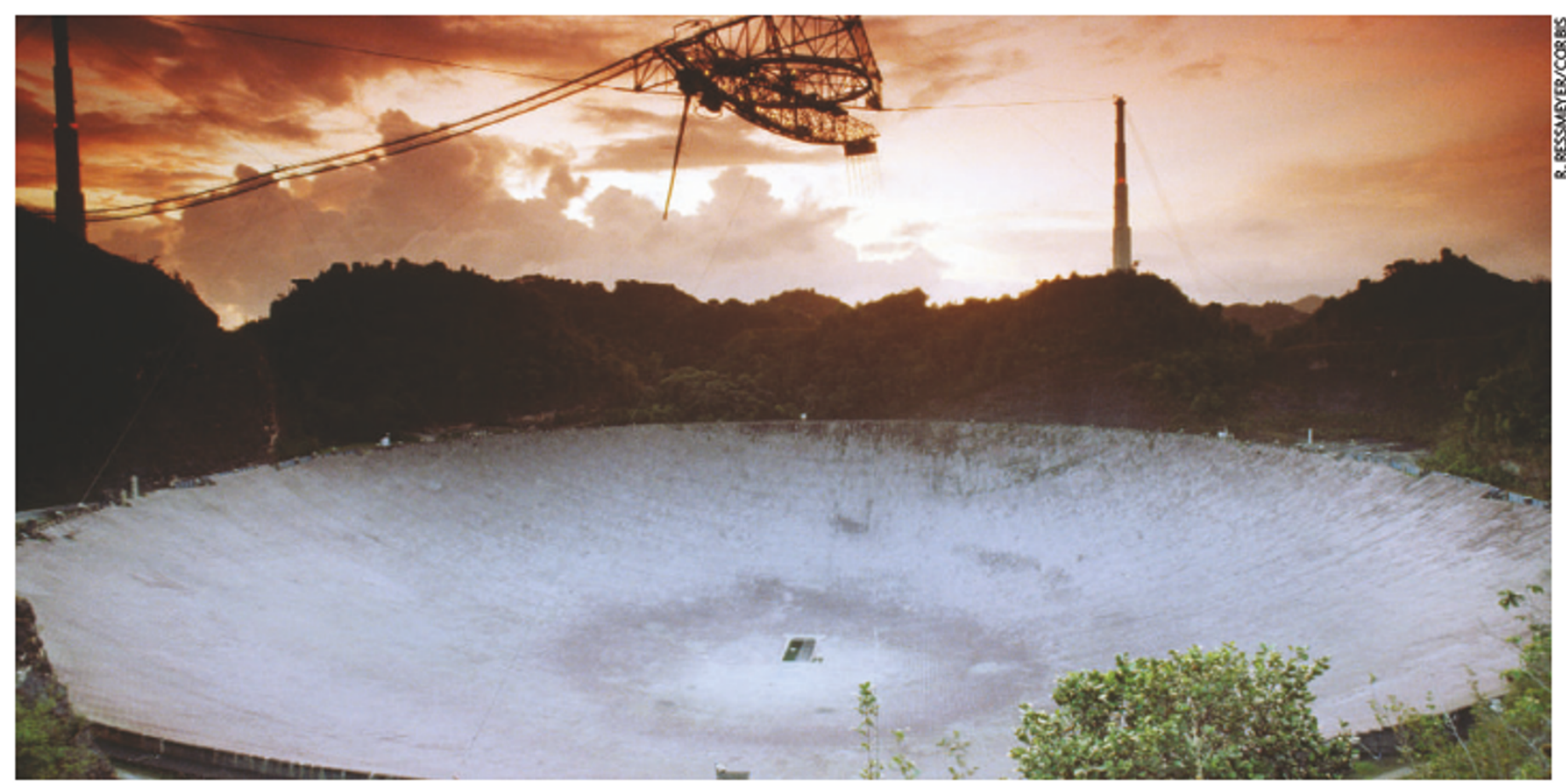

Lights out: the Arecibo radio telescope in Puerto Rico is one of several facilities that could face budget cuts to secure funding for larger projects.

\section{Telescope is no longer dish of the day}

With a history of nurturing Nobel prizewinners and landing star roles in Hollywood films, the Arecibo radio telescope in Puerto Rico has worked its way into both scientific and popular cultures. Since 1963, the telescope's 70,000square-metre collecting area has been used for everything from characterizing pulsars to the finale of the James Bond film Goldeneye.

But, according to a review committee, the facility should close if it cannot find outside financial support by 2011. The committee delivered its verdict to the Division of Astronomical Sciences of the US National Science Foundation (NSF) on 3 November. Arecibo is not the only facility facing the axe - the committee also recommended that the continent-wide Very Large Baseline Array should seek external funding or face closure. In addition, funding for operations by the National Solar Observatory (NSO) on Kitt Peak near Tucson, Arizona, and Sacramento Peak in Sunspot, New Mexico, could end if the planned Advanced Technology Solar Telescope receives final approval.

None of the proposals is a death sentence; the NSF will consider the recommendations over the next couple of months. But the observatories in question may now turn to universities and foreign collaborators for funding. "Nobody's out here nailing plywood over the windows," says David Dooling, a spokesman for the NSO. "We think there are still a lot of years left."
But the committee's findings highlight a growing problem in US ground-based astronomy: the expanding appetite for new telescopes doesn't leave much room for the continuing operations budgets of older telescopes such as Arecibo. Among other new projects, the NSF will pay $\$ 500$ million for its share of the Atacama Large Millimeter Array, a 50-antenna cluster scheduled to be operational in Chile by 2013. The foundation also aims toparticipate in the Square Kilometre Array, an international effort likely to cost more than a billion dollars in total.

${ }^{\alpha}$ To make room for the new you have to trim some of the old," says Michael Turner, an astrophysicist at the University of Chicago. "If you keep building new facilities without closing old facilities, eventually the operations budget eats your entire budget." To that end, the committee - led by astronomer Roger Blandford of Stanford University - was asked in October 2005 to find ways of saving $\$ 30$ million in the NSF's astronomy facilities budget by 2011.

The committee report recommends that astronomical facilities "plan for a natural life cycle ending, in every case, with closure".

Turner says that ground-based astronomy has so far been spared difficult choices over which facilities to close, unlike the decommissioning of space telescopes: ${ }^{\alpha}$ This is much more difficult to do on the ground because space facilities fall from the sky and don't live in anyone's congressional district." (This might surprise Maryland Senator Barbara Mikulski, whose support of life-extending missions to the Hubble Space Telescope has been appreciated at the Space Telescope Science Institute in Baltimore, Maryland.)

Donald Campbell, an astronomer at Cornell University in Ithaca, New York, who has used Arecibo to study planets and the Moon, says that the telescope's potential closure is particularly painful in light of major upgrades, the latest in 1997. If Arecibo closes, he says he will have to rely entirely on the less-sensitive Goldstone radar telescope operated byNASA in California's Mojave Desert. 'It's a great shame that the choice has to come down to closing facilities that are unique, he says.

Observers overseas agree. "It's a pity that they were under pressure to make these cuts in facilities that were still productive by the standards of other scientific facilities," says Martin Rees, a cosmologist at the University of Cambridge, UK, and president of the Royal Society. Rees says that Britain faced a similar decision when it elected to join the European Southern Observatory, and funding pressures forced the country to close some smaller telescopes.

Heidi Ledford 